\title{
ENVEJECIMIENTO, DEPENDENCIA Y CUIDADOS. RETOS SOCIALES Y RETOS ASISTENCIALES
}

DOLORS COMAS-D'ARGEMIR MONTSERRAT SORONELLAS-MASDEU Universitat Rovira i Virgili

Debemos dejar de ver la vejez como una catástrofe, porque no lo es. El incremento de la longevidad es un triunfo histórico, conseguido gracias a unas mejores condiciones de vida y a unos sistemas sanitarios que protegen a la población en caso de enfermedad, y ha de ser percibido, pues, como un logro del progreso social y de la democratización de la supervivencia y no como una catástrofe. Y desde este foco hay que afrontar un futuro no demasiado lejano (aunque el coronavirus ha impactado sobre estas previsiones) en que el número de personas adultas y de personas mayores será mucho mayor que el número de jóvenes, algo que las migraciones o un incremento de la natalidad pueden mitigar, pero no frenar.

Aunque la vejez sea percibida de forma negativa como fruto de un edadismo que resalta aspectos como el impacto en los costes sanitarios y sociales, las situaciones de soledad o el rechazo a la decrepitud de los cuerpos, hay que reconocer que en las últimas décadas el proceso de envejecimiento se ha transformado de forma espectacular y ha contribuido a modificar aspectos esenciales de la vida social. La etapa de la vejez se 
ha dilatado en el tiempo y supone una percepción nueva del curso vital. Actualmente, una mujer española de 70 años tiene la misma esperanza de vida que tenía una mujer española de 56 años en 1960. Esto entraña cambios en la definición de qué es una persona mayor, así como en la representación de las trayectorias biográficas. Mary Catherine Bateson (2013) considera que el aumento de la longevidad no ha contribuido a extender la etapa de la vejez, sino que ha creado una nueva etapa, que ella denomina «segunda edad adulta». Y a la «tercera edad» se añade una cuarta e, incluso, una quinta edad. La presencia simultánea de cuatro generaciones en las familias constituye una verdadera revolución en las dinámicas sociales y familiares que ha hecho nacer no solo unas relaciones intergeneracionales inéditas, sino también una transformación de los roles de género, de las necesidades de cuidados y de las políticas públicas dirigidas a las personas mayores (Attias-Donfut y Segalen, 2007).

Todas estas transformaciones hacen que, en el contexto de las sociedades europeas contemporáneas, se pueda hablar de «nuevos envejecimientos» (Comas d'Argemir y Roigé, 2018). Y es que esta «segunda edad adulta» se caracteriza por su gran vitalidad y elevada participación social y política, lo que rompe los estereotipos asociados a la vejez. Las personas mayores no representan las supervivencias de un pasado al cual se aferran, sino que son sujetos activos en la transformación de las sociedades. No se pueden identificar solamente (ni principalmente) con personas en situación de dependencia, sino como sujetos que crean un nuevo tipo de ciudadanía activa que influye en las decisiones sociales y políticas y que cada vez lo hará más como fruto de su mayor presencia en número y en protagonismo. En el contexto familiar, por otra parte, se ha incrementado el rol social, simbólico y práctico de las personas mayores, pues, como ocurre en el conjunto de la sociedad, tienen mayor presencia en unas familias extensas que no solo no han desaparecido, sino que, por el contrario, se han consolidado (Attias-Donfut et al., 2002). Actualmente, la estructura intergeneracional es más compleja. Hay más generaciones que pueden coexistir durante un período de tiempo más largo y cada individuo puede tener más de dos roles o posiciones generacionales en el curso de vida, incluso de forma simultánea. Así, una persona puede ser a 
la vez abuela, madre e hija, y especialmente el estatus de hija puede alargarse durante muchos años (Saraceno, 2016). Por otro lado, las personas mayores tienen también un rol activo en las transformaciones familiares, pues en su trayecto vital pueden haber experimentado las evoluciones provocadas por el divorcio, la recomposición de las parejas o la pluriparentalidad (Roigé y Soronellas, 2018).

Evidentemente, el perfil de las personas mayores varía mucho como efecto de las desigualdades sociales. El tan estimado envejecimiento activo es más fácil de conseguir cuando se dispone de recursos económicos y culturales, así como de un amplio abanico de redes familiares y sociales. Los sectores de población más humildes envejecen en peores condiciones de salud, y la esperanza de vida es más baja que entre los sectores más adinerados. Por otra parte, no se envejece igual siendo hombre o siendo mujer. El ejercicio de los roles de género a lo largo de la vida establece desigualdades que se reproducen y acrecientan en la vejez (Ramos, 2017). También las transformaciones de los cuerpos se perciben de forma diferente, de manera que para las mujeres es un imperativo tejer estrategias para disimular los signos del envejecimiento y evitar la invisibilidad social asociada a la vejez, que, en el caso de los hombres, se expresa de forma bien distinta (Sontag, 1972). Cabe mencionar, finalmente, las diferencias en la forma de envejecer entre las personas que no residen en sus países de origen y que tienen el dilema de permanecer o de retornar.

Estas cuestiones relacionadas con el proceso de envejecimiento y la vejez aparecen en los artículos de este número monográfico. Pero hay otro tema importante que aparece también, incluso con mayor extensión. Se trata de los cuidados hacia las personas mayores que han perdido su capacidad de autonomía y han entrado en situación de dependencia, lo que implica necesitar de terceras personas para realizar actividades básicas de la vida diaria. El análisis de los cuidados en la etapa del final de la vida constituye un punto crítico para comprender cómo este incremento de la longevidad modela las experiencias y significados del cuidado, moviliza agentes y recursos para llevarlo a cabo y constituye un punto de interconexión entre la economía moral y la economía política (Buch, 2015). 
La familia es un agente esencial en la provisión de cuidados relacionados con la dependencia. A pesar de que, tal como señalan Mary Daly y Jane Lewis (2000) cuando presentan el concepto de cuidado social, el Estado y el mercado proporcionan también recursos de cuidado, la familia sigue recibiendo de la sociedad el encargo de cuidar de sus miembros. Mejor dicho, se considera que tiene la responsabilidad de hacerlo. Y dentro de la familia son las mujeres las encargadas de realizarlo, como un mandato derivado de sus roles de género y de parentesco (Glenn, 2010). Es así en la vida diaria, cotidiana, en que se practica el cuidado propio y el de las personas del entorno familiar inmediato. Pero el cuidado toma su visibilidad cuando se configuran necesidades especiales que derivan de las situaciones de crianza (cuidado de niños y niñas) o del cuidado hacia personas mayores y dependientes. Es en este marco donde el cuidado desborda el marco familiar para pasar a ser una cuestión social y política.

Las familias jerarquizan a sus miembros a partir de una estructura según la cual el género, la generación y la posición genealógica se combinan para distribuir las responsabilidades del cuidado de sus miembros. El compromiso del cuidado lo reciben prioritariamente las mujeres de la familia, que lo asumen y naturalizan como un deber propio, en su calidad de madres, esposas o hijas. Cuidar hace género y hace también parentesco (Comas d'Argemir y Soronellas, 2019). El parentesco se construye y reproduce a través del ejercicio del cuidado y las formas de cuidar y ser cuidado configuran el parentesco y estructuran las relaciones entre los parientes (Carsten, 2000).

En las prácticas de cuidado conviven tres regímenes morales: el don, la reciprocidad y la mercancía, que están fuertemente generizados (Comas d'Argemir, 2017). Los cuidados familiares son percibidos como don y como obligación moral para con sus parientes; también como un compromiso que se negocia con la persona cuidada y con el resto de familiares involucrados en el trabajo de cuidar. Pagar a otras personas para que se encarguen de cuidar se inscribe en el marco de estas obligaciones morales, para resolver las dificultades de dedicarse enteramente a la actividad de cuidar. Finalmente, el cuidado es percibido también como un espacio donde puede surgir el conflicto y donde interaccionan materiali- 
dades que a menudo quedan ocultas bajo la emocionalidad que envuelve el don del cuidado.

La arquitectura del cuidado está construida a partir de desigualdades de género y de desigualdades sociales. La vida de las mujeres está atravesada por el cuidado, lo que impacta en sus trayectorias laborales y sociales, constituyendo un elemento de discriminación y desposesión. Por otro lado, las personas con pocos recursos envejecen peor, tienen más necesidades de cuidados y menos recursos materiales para resolverlas, lo que obliga mucho más a las mujeres de la familia. Donde las políticas públicas para atender los cuidados son débiles, como sucede en España, se hacen más necesarios este altruismo obligatorio que practican las mujeres y la solidaridad intergeneracional. Esta insuficiencia en recursos públicos obliga, a su vez, a las familias a recurrir al trabajo de mujeres migrantes, con salarios precarios y pésimas condiciones laborales (Offenhenden, 2017). Es esta otra expresión de cómo el cuidado está configurado desde ejes de desigualdad. Por esto indicamos la necesidad de democratizar los cuidados. A ello nos referiremos más adelante.

En la literatura académica se ha acuñado la expresión «crisis de los cuidados» para referirse a una situación paradoxal, y que está llegando a una situación límite, y es el hecho de que, mientras se incrementan las necesidades de cuidados (especialmente los relacionados con la vejez), disminuyen las posibilidades de cuidar (Pérez-Orozco, 2006). Esta crisis de los cuidados se origina por la intersección entre unos cambios demográficos y unos cambios sociales y culturales de gran magnitud.

Estamos a nivel mundial en un momento de transición demográfica: hemos pasado de un régimen caracterizado por una alta mortalidad de tipo catastrófico y una elevada fecundidad a otro en que se invierten las variables anteriores: reducción de la mortalidad y disminución de la natalidad. Esta dinámica conforma el envejecimiento de nuestras sociedades: vivimos más años y hacemos menos hijos. Resultado: nuestras sociedades envejecen. Dos son las razones más comúnmente atribuidas a este proceso.

El descenso de la fecundidad se ha producido en prácticamente todos los países del mundo, de manera que en el transcurso de la segunda mi- 
tad del siglo xx las tasas de fecundidad se sitúan por debajo de las tasas de reemplazo de la población. Así sucede en países tan diversos como Estados Unidos, Chile, Brasil, Alemania, China, Japón o Rusia. España tiene una de las tasas de fecundidad más bajas del mundo ( 1,3 hijos por mujer). Solo los países africanos están experimentando actualmente un crecimiento demográfico. Por otro lado, se ha producido una drástica reducción de la mortalidad en todas las etapas de la vida, especialmente entre las personas de más de 55 años, lo que comporta una mayor proporción de personas con más de 80 años y un notable incremento de la esperanza de vida al nacer. Si nos situamos en el caso de España, por ejemplo, en 1960 la esperanza de vida de las mujeres era de 71,7 años, mientras que en 2020 era de 85,1. En el caso de los hombres era en 1960 de 66,7 años, y en el 2020 de 79,7 (13,4 y 13 años más, respectivamente).

Estos cambios demográficos tienen consecuencias para las necesidades de cuidados. Tenemos, por un lado, una infancia que disminuye en número, aunque, al mismo tiempo, se incrementan las exigencias en su cuidado (alimentación, salud, educación, socialización, atención directa). Además, hay también necesidades de cuidado de la denominada «vejez-frágil», es decir, de personas mayores que no pueden valerse por sí mismas. Finalmente, deben añadirse las situaciones de discapacidad o de limitación derivadas de enfermedades crónicas y alteraciones congénitas, así como de la siniestralidad vial y laboral. Son realidades que conectamos a través de lo que llamamos cuidados, pero que cada una tiene su especificidad y dentro de cada una hay, además, singularidades.

En paralelo a estos cambios demográficos se han producido cambios sociales y familiares muy importantes. El más relevante es la participación laboral de las mujeres de clase media (las de clase obrera siempre la han ejercido) y la consecuente generalización de las familias de doble salario, en que tanto los hombres como las mujeres aportan ingresos para el sustento familiar. Esto ha ido acompañado en el caso de España de un mayor acceso a la educación por parte de la población y de una movilidad social ascendente a través de los estudios. En consecuencia, actualmente las mujeres tienen menos disponibilidad para cuidar (Comas d'Argemir, 1995). La figura del ama de casa, dedicada a cuidar de sus familiares, prác- 
ticamente ha desaparecido y queda reducida a las generaciones mayores, y frecuentemente han sido las mujeres mayores quienes han estimulado a sus hijas a estudiar, a tener una vida propia y no estar atadas a los cuidados (Conlon et al., 2014). También las familias han experimentado cambios sustanciales, a partir de la presencia de divorcios, familias recompuestas, familias monoparentales, y la diversidad de formas de convivencia. Unas familias a menudo fragmentadas por la lógica de la vida urbana y también por las migraciones.

A pesar de estos cambios en la familia y en la vida de las mujeres, el modelo de cuidados se sigue sustentando idealmente en la familia y en la figura de la mujer como pilar básico. Un modelo que ya no existe. Los hombres, por su parte, han incrementado su participación en las actividades de crianza, pero apenas se implican en los cuidados de personas mayores y dependientes (Comas d'Argemir y Soronellas, 2019). Donde las políticas públicas son débiles, como sucede en el caso de España o de Italia, por ejemplo, la familia tiene que hacerse cargo de unos cuidados que cada vez son más difíciles de resolver, ya que en el caso de la vejez aumenta el tiempo en que se requieren cuidados y estos, a su vez, son de mayor complejidad e intensidad. En estas condiciones, la crisis de los cuidados está servida.

En el congreso internacional que celebramos en Tarragona en septiembre de 2019, con el título El cuidado de mayores y dependientes. Promoviendo la igualdad de género y la justicia social, organizado conjuntamente con la Universidad de Barcelona, insistimos en la urgente necesidad de democratizar los cuidados. Señalábamos que los cuidados estaban provistos desproporcionadamente por la familia y por las mujeres y que se requería la implicación de los hombres y un mayor compromiso público y comunitario.

Democratizar los cuidados supone plantear una organización social del cuidado que favorezca tanto a las personas que los reciben como a quienes los proporcionan. Surge de una propuesta de Sandra Ezquerra y Elba Mansilla (2018) que asume el Ayuntamiento de Barcelona como guía de la intervención social en este tema y que hemos empezado a utilizar en el marco académico como referencia y propuesta política. La demo- 
cratización de los cuidados pasa por los siguientes ejes: 1) promover el reconocimiento del cuidado y de su centralidad; 2) socializar las responsabilidades del cuidado; 3) repartir el cuidado entre hombres y mujeres, para eliminar la (mal)división sexual del trabajo, y 4) tener en cuenta los derechos y las demandas de las personas receptoras de cuidados, en función de su ciudadanía y no solo como consumidores. Cada una de estas dimensiones es un paso positivo hacia la democratización de los cuidados, pero el avance de solo una de ellas constituye una democratización parcial o incluso un retroceso. Por ejemplo, si no se dan de forma simultánea una socialización del cuidado y un reconocimiento social de este, esta socialización podría darse en una dirección mercantilizadora y perjudicar el derecho al cuidado de los colectivos pobres. Por esto las cuatro dimensiones están interrelacionadas.

Cuando organizamos el congreso y cuando lo realizamos (septiembre de 2019), estábamos muy lejos de conocer que una pandemia tan terrible como el coronavirus cambiaría nuestro mundo de forma tan drástica y repentina. Lo que entonces nos parecía pertinente, urgente, imprescindible de tratar (como es el cuidado a mayores y dependientes), hoy está de plena actualidad. La pandemia ha revelado la fragilidad del sistema de cuidados tal como está organizado actualmente. Y ha revelado también que se trata de una cuestión social y política, no de una mera cuestión individual o encerrada en las familias.

Una parte de las contribuciones a este congreso está recogida en este número monográfico que tiene en los cuidados su hilo conductor. En conjunto, los textos se adentran en los ejes que acabamos de presentar, el envejecimiento de la población y el reto social y político que supone afrontar este proceso, y el desafío de organizar los cuidados de forma justa y sostenible. Los datos y reflexiones aportados por los artículos de este monográfico sitúan el foco en los diferentes agentes proveedores de cuidados: las familias, el Estado y las políticas públicas, el mercado y las comunidades organizadas para cuidar. Abre el monográfico el artículo de Albert Julià, titulado «Factors associats als tipus de cures domiciliàries de les persones en situació de dependència funcional a Barcelona», donde nos presenta los datos de una encuesta realizada en la ciudad de Barce- 
lona en 2018 para conocer, por un lado, cuáles eran las necesidades de cuidado de las personas en situación de dependencia funcional, y, por el otro, cuáles eran los agentes provisores de los cuidados. Los datos resultan relevantes porque no existen en España datos oficiales sobre cuidados más allá de los que recoge el Sistema de Atención a la Dependencia (SAAD) sobre las solicitudes y las resoluciones de la ayuda a la dependencia de la LAPAD. Los datos presentados por Julià muestran la desigualdad de género derivada de la organización de los trabajos de cuidado, puesto que siguen siendo trabajos feminizados, tanto en el ámbito familiar como en el profesional, y que las familias siguen siendo agentes esenciales de cuidados. La debilidad de las políticas públicas propicia el cuidado familiar y crea situaciones de desigualdad, puesto que las personas sin recursos económicos no pueden acceder al mercado para proveerse de los recursos de cuidado necesarios. Julià nos alerta también sobre las situaciones de vejez en soledad y precariedad económica como las de mayor vulnerabilidad y dependencia respecto a unos recursos públicos a todas luces insuficientes para cubrir sus necesidades de cuidado. El Estado, nos dice, tiene la responsabilidad de atender estas situaciones y de liberar a las familias de la responsabilidad total del cuidado. También Patricia Estefanía Celi se refiere a la responsabilidad de las familias y del Estado en el cuidado de personas dependientes, en este caso en Ecuador. Celi ha estudiado la percepción sobre la responsabilidad compartida familia-Estado entre cuidadores familiares y personas responsables de formular y ejecutar las políticas de cuidados y concluye que los cuidados familiares están ampliamente normalizados tanto en las familias (las mujeres) que los proveen, las cuales se sienten moralmente responsables de cuidar, como en el Estado, que no despliega políticas de bienestar más que para atender a personas en situación de exclusión social. Por todo ello, concluye Celi, mientras los cuidados no sean asumidos como asunto público, es difícil avanzar en la superación de la división de roles entre hombres y mujeres y romper las actuales estructuras injustas de desigualdad y poder que organizan los cuidados.

Continuando con el papel del Estado, o su ausencia, en el desarrollo de políticas de bienestar y cuidados, Magdalena Zegarra, en «Biopolitically 
(un)caring: Sustaining bodies and shaping care in precarious environments», nos acerca al papel de las instituciones en la gestión de los cuidados de personas mayores, solas y sin recursos en un asilo de la ciudad de Lima, en Perú (el albergue de la Merced). Después de un extenso trabajo etnográfico en esta institución, Zegarra sostiene que el cuidado es provisto esencialmente como una práctica de control biopolítico de los cuerpos y de las vidas de las personas mayores residentes. Nos relata cuidados circunscritos al límite de la mera existencia y del maltrato, que satisfacen en sentido estricto las necesidades biológicas de las personas, pero que no satisfacen sus necesidades emocionales y que no garantizan el bienestar de las personas «cuidadas» ni el de las personas cuidadoras, que se encuentran condicionadas por el contexto de precariedad material y moral en el que deben realizar su trabajo. A la dimensión emocional del cuidado se refiere también Carmina Puig en su artículo «Los cuidados invisibles. Afectividad y acciones intangibles de los cuidados». La autora sostiene que las dimensiones emocionales y morales deben ser tenidas en cuenta para amplificar la mirada hacia los cuidados invisibles con el fin de dejar en evidencia los elementos intangibles del cuidado. Puig fija su atención en la experiencia de cuidar y en el impacto que la relación de cuidado tiene sobre la persona cuidadora y la cuidada. El cuidado facilita el descubrimiento de la vulnerabilidad propia y ajena y es un arma poderosa en la medida que hace evidente la interdependencia. Las dimensiones física y operativa y la emocional no pueden ser identificadas separadamente, las dos confluyen en el compromiso común del cuidado.

Kaori Miyachi y Ken Masuda, en su texto «A preparatory Study of the Care for Old Women in Rural Kenya», analizan el proceso de envejecimiento y la organización de los cuidados de mujeres mayores en el condado de Kwale, en la Kenia rural. Masuda y Miyachi dibujan una sociedad rural en que la provisión de cuidados está completamente en manos de las mujeres y de las familias, con una total ausencia del Estado en la provisión del cuidado tanto médico como social. Una construcción de la organización social del cuidado basada en el género y en el parentesco según la cual son las mujeres, hijas y nueras quienes tienen la responsabilidad del cuidado. Los cambios sociales provocados por el éxodo rural y la mi- 
gración han interrumpido los ciclos de retorno del compromiso del cuidado, por lo que muchas de las mujeres del estudio envejecen solas o con escasa red de apoyo. Herminia Gonzálvez y Menara Guizardi nos sitúan, en cambio, ante mujeres que se organizan en redes comunitarias para proporcionarse cuidados mutuos en Chile. En su artículo «Las mujeres mayores, los cuidados y los clubes: negociaciones intersubjetivas de una etnografía feminista», las autoras abordan el cuidado y las intersubjetividades derivadas de este desde una etnografía feminista que valoriza las agencias de las mujeres en la creación de espacios comunitarios para el cuidado recíproco. El texto se adentra también en una reflexión relevante sobre experiencias personales de género y de cuidados que contribuyeron a dar sentido a la selección del objeto de investigación: el envejecimiento femenino. Después de una muy pertinente reflexión sobre la metodología aplicada en el proyecto, las autoras nos proponen ir más allá de los argumentos dicotómicos para adentrarnos en el cuidado como articulador de redes femeninas y como «la forma en que las mujeres construyen y, a la vez, desafían las fronteras del patriarcado».

También desde un enfoque de género, pero focalizando en los hombres que cuidan, en nuestro texto «Hombres que deciden cuidar a personas adultas dependientes en el contexto familiar, género y parentesco en transformación», analizamos la situación de hombres que han tomado la decisión de dejar sus actividades profesionales para dedicarse al cuidado de esposas e hijos adultos en situación de dependencia. Aparece de nuevo la familia como un agente esencial de cuidado, aunque en este caso ejercido por hombres, algo singular en un contexto de organización social del cuidado, injusta y desproporcionadamente ejercido por las mujeres. Precisamente por ello estos hombres, maridos y padres que toman la decisión de cuidar tensionan las prescripciones de las categorías del género y del parentesco en la organización social del cuidado.

Sobre el envejecimiento y la alta incidencia de enfermedades asociadas al deterioro cognitivo versa el artículo «A relevância do desenvolvimento local para a promoção do florescimento humano na doença de Alzheimer (Portugal)», de Joan Carrilho da Silva. La autora parte del sobreenvejecimiento de la población y del incremento del número de per- 
sonas afectadas por la enfermedad de Alzheimer y revisa algunas de las políticas públicas destinadas a la mejora de la calidad de vida de las personas afectadas por la enfermedad. Tomando en cuenta la diversidad de actores que interviene en el cuidado de las personas enfermas, Carrilho persigue entender cómo cada uno de ellos percibe una enfermedad socialmente estigmatizada y cómo actúa sobre ella y sobre las distintas dimensiones del bienestar de las personas cuidadas. Carrilho propone relacionar los diferentes actores en un proyecto de desarrollo local de alcance comunitario que sostenga una visión integrada y multidisciplinar sobre la atención a los procesos de envejecimiento y al alzhéimer.

La toma en consideración del entorno local-comunitario de las situaciones de cuidado también es protagonista en el texto de Teresa Vicente y Yayza Pérez Alonso, titulado «Personas mayores como posibilidad de inserción sociolaboral en el barrio del Cabanyal-Canyamelar-Cap de França, de Valencia». Las autoras nos sitúan de nuevo en el contexto del Estado y de la política pública para mostrarnos el cuidado remunerado como una estrategia de inclusión social de colectivos vulnerables. La formación ocupacional en materia de cuidados puede tener un papel clave en la inserción de personas en riesgo de exclusión y que, por su edad o por sus déficits formativos, tienen dificultades de inserción en el mercado de trabajo. De este modo confluyen diferentes intereses de las políticas sociales: por un lado, la capacitación profesional de personas con dificultades de inserción laboral, y, por otro, la mejora del servicio de atención a la dependencia, especialmente de las personas mayores.

En su conjunto, los textos de este monográfico constituyen una buena compilación que revisa algunas de las más relevantes dimensiones del estado actual de la investigación sobre sobre envejecimiento y cuidados de personas adultas dependientes. Deseamos que resulte de utilidad a las personas que están investigando sobre estas cuestiones, y que sea también una invitación a seguir reflexionando sobre el cómo y el porqué de la organización de los cuidados, sin olvidar la necesaria reivindicación del reconocimiento político del lugar esencial que ocupan los cuidados en la sociedad. 


\section{Bibliografía}

Attias-Donfut, C.; Lapierre, N. y Segalen, M. (2002). Le nouvel esprit de famille. Paris: Odile Jacob.

Attias-Donfut, C. y Segalen, M. (2007). Grands-parents : la famille à travers les générations. Paris: Odile Jacob.

Bateson, M. C. (2013). «Changes in the life course. Stregths and Stages». En C. Lynch y J. Danely (eds.). Transitions and transformations. Cultural perspectives of aging and the life course (pp. 21-34). New York; Oxford: Berghahn Books.

Buch, E. D. (2015). "Anthropology of aging and care». Annual Review of Anthropology, 44 (2), 277-293.

CARSTEN, J. (ed.) (2000). Cultures of relatedness: New approaches to the study of kinship. Cambridge: Cambridge University Press.

Comas-d’Argemir, D. (1995). Trabajo, género y cultura. La construcción de desigualdades entre hombres y mujeres. Barcelona: Icaria.

Comas-D’Argemir, D. (2017). «El don y la reciprocidad tienen género: las bases morales de los cuidados». Quaderns-e de l'Institut Català d'Antropologia , 22 (2), 17-32.

Comas-d'Argemir, D. y Roigé, X. (2018). «Between family and state: The new faces of ageing in Europe». Ethnologie francaise, 171, 389-400.

Comas-d'Argemir, D. y Soronellas-Masdeu, M. (2019). Men as carers in long-term caring: Doing gender and doing Kinship. Journal of Family Issues, 40 (3), 315-339.

Conlon, C.; Virpi, T.; Gemma, C. y Thomas, S. (2014). «Women (re) negotiating care across family generations: Intersections of gender and socioeconomic status». Gender \& Society, 8, 729-751.

DALY, M. y LEWIS, J. (2000). «The concept of social care and the analysis of contemporary welfare states». The British Journal of Sociology, 51 (2), 281-298.

Ezquerra, S. y Mansilla, E. (2018). Economia de les cures i politica municipal: cap a una democratització de la cura a la ciutat de Barcelona. Barcelona: Ajuntament de Barcelona, Universitat de Vic, La Ciutat Invisible.

FEDERICI, S. (2013). Revolución en punto cero. Trabajo doméstico, reproducción y luchas feministas. Madrid: Traficantes de Sueños. 
FraSER, N. (1997). «Después del salario familiar. Un experimento conceptual postindustrial». En Iustitia Interrupta. Reflexiones críticas desde la posición «postsocialista». Bogotá: Siglo del Hombre Editores, Universidad de los Andes.

GLENN, E. N. (2010). Forced to care: Coercion and caregiving in America. Harvard University Press.

Offenhenden, M. (2017). Si hay que romperse una, se rompe. El trabajo del hogary la reproducción social estratificada (tesis doctoral). Universitat Rovira i Virgili.

Pérez-Orozco, A. (2006). «Amenaza tormenta: la crisis de los cuidados y la reorganización del sistema económico.» Revista de Economía Crítica, 5, 7-37.

Pérez-Orozco, A. (2014). Subversión feminista de la economía. Aportes para un debate sobre el conflicto capital-vida. Madrid: Traficantes de Sueños.

Ramos, M. (2017). Envejecer siendo mujer. Dificultades, oportunidades y retos. Barcelona: Bellaterra.

Roigé, X. y Soronellas-Masdeu, M. (2018). «Vieillissement, divorce et recomposition familiale. Nouveaux défis dans les relations d'aide aux personnes âgées». Ethnologie française, 171, 465-478.

SARACENo, C. (2016). Coppie e famiglie. Non è questione di natura. Milano: Universale Economica Feltrinelli; SAGI.

SonTAG, S. (1972). «The double standard of aging». The Saturday Review, septiembre, 23, 29-38. 